\title{
SCCmec typing and Panton-Valentine leukocidin occurrence in methicillin resistant Staphylococcus aureus isolates from clinical samples of Ahvaz, southwest of Iran
}

\author{
Nikou Bahrami, Hossein Motamedi, Seyyedeh Elham Reza Tofighi, Mohammad Reza Akhoond \\ Shahid Chamran University of Ahvaz, Islamic Republic of Iran
}

\begin{abstract}
Summary
Resistance to methicillin in methicillin resistant Staphylococcus aureus (MRSA) is dependent on mecA gene located on staphylococcal cassette chromosome (SCC). Both SCCmec type and Panton-Valentine leukocidin (PVL) affect $S$. aureus pathogenicity. Aim of this study was to investigate the prevalence of SCCmec $A$ types and $p v l$ genes among MRSA isolates from inpatients. During this cross-sectional study on 100 clinical isolates, following antibiotic susceptibility test, screening of mecA and $p v l$ genes, as well as SCCmec typing, was done in a multiplex PCR technique. From the studied samples, 58 isolates were recognized as MRSA. The frequency of mecA and $p v l$ was $58 \%$ and $4 \%$, respectively. All of the MRSA were resistant to cefoxitin and had the highest sensitivity to chloramphenicol. The majority $(77.5 \%)$ of MRSA was originated from wound samples. The SCCmec III was the most frequent type $(22.4 \%)$ in these samples. The $p v l$ positive isolates were from SCCmec IVb and $\mathrm{V}$, thus meaning they
\end{abstract}

\footnotetext{
Correspondence: Hossein Motamedi, Department of Biology, Faculty of Sciences, Shahid Chamran University of Ahvaz, Islamic Republic of Iran.

E-mail: hhmotamedi@yahoo.com
}

Key words: SCCmec; Methicillin resistant Staphylococcus aureus; SCCmec typing; Panton-Valentine leukocidin.

Acknowledgments: the authors wish to thank the research Vice Chancellor of Shahid Chamran University of Ahvaz for providing research grant (Grant No. 96/3/02/32860), and also MSc. thesis grant.

Contributions: the authors contributed equally.

Conflict of interest: the authors declare no potential conflict of interest.

Funding: none.

Received for publication: 23 January 2019.

Revision received: 7 April 2019.

Accepted for publication: 13 September 2019.

${ }^{\circ}$ Copyright: the Author(s), 2019

Licensee PAGEPress, Italy

Microbiologia Medica 2019; 34:8050

doi:10.4081/mm.2019.8050

This article is distributed under the terms of the Creative Commons Attribution Noncommercial License (by-nc 4.0) which permits any noncommercial use, distribution, and reproduction in any medium, provided the original author(s) and source are credited. are from CA-MRSA. These results show a high prevalence of MRSA in the studied region and a widespread prevalence of SCCmec I-V types. Furthermore, high prevalence of SCCmec III indicates the prevalence of multidrug resistant MRSA. This finding is a serious alarm for medical health care practitioners for the correct use of antibiotics in order to limit the spread of multidrug resistant strains. In addition, with regard to life threatening infections caused by $p v l$ harbouring strains, early diagnosis and treatment of infections caused by these isolates should be mandatory.

\section{Introduction}

Staphylococcus aureus (S. aureus) is one of the most important agents of hospital acquired infections that causes self-limiting to life-threatening opportunistic infections $(1,2)$. The methicillin resistant $S$. aureus (MRSA) is one of the main causes of community and hospital acquired infections that can lead to treatment failure (1) and mortality (3). Following appearance of penicillin resistant strains, methicillin was introduced for treatment of $S$. aureus infections but in 1961, just one year following its introduction in the clinical practice, MRSA strains were reported to be present in European hospitals. The emergence of such strains was then reported from other countries such as Canada, North America and Australia. This resistance is due to the acquisition of $m e c A$ gene $(2.1 \mathrm{kbp})$ that encodes $\mathrm{PBP}_{2 \mathrm{a}}$ and can move between bacterial strains through a large movable chromosomal cassette, namely SCCmec and inserted at a specific site as $\operatorname{Orfx}$. $\mathrm{PBP}_{2 \mathrm{a}}$ has a low affinity for $\beta$-lactam antibiotics (1). The SCCmec have two essential element cassettes including $\mathrm{ccr}$ gene complex and mec gene complex (4). Until today, 11 main types of SCCmec (I-XI) have been identified and the difference of SCCmec type is applied as a clue for differentiating MRSA clones. Hospital acquired MRSA (HA-MRSA) is mainly found in old patients with predisposing factors such as catheter use, while community acquired MRSA (CA-MRSA) mainly affects younger people without predisposing factors. CA-MRSA is usually sensitive to $\beta$-lactam antibiotics and harbour SCCmec type IV, V or VI while HA-MRSA strains show multidrug resistance with SCCmec type I, II or III (5). Therefore, typing of MRSA isolates can reveal the genetic origin of them and provides suggestions for controlling hospital infections in order to prevent cross contaminations (6). Nearly, all $S$. aureus isolates produce different toxins and enzymes that are essential in their pathogenicity. Panton-Valentine leukocidin (PVL) is a cytolysin and a virulent factor of $S$. aureus. This cytotoxin is specific for leukocytes and two ORFs (Luks-PV and Lukf-PV) encode it. The $p v l$ positive strains are commonly involved in skin and mucosal membrane infections, necrotizing pneumonia, urinary infections and endocarditis $(7,8,9)$. The presence of $p v l$ gene is 
regarded as a marker for MRSA (4). The $p v l$ harboring MRSA usually has SCCmec types IV and V and is related with CA-MRSA (10). Regarding the role that $\mathrm{SCCmec}$ plays in resistance and pathogenicity and the relation between virulent factors and SCCmec type, typing of MRSA based on SCCmec can be a useful and practical method for epidemiological study, and a key element for revealing their origin and distribution in different regions. The aim of present study was to investigate the prevalence of $p v l$ gene and SCCmec type among clinical $S$. aureus isolates from Razi Hospital of Ahvaz, Iran.

\section{Materials and Methods}

During a 9-month period in a cross-sectional study, S. aureus isolates from blood, wound, tracheal secretions, urine and pus samples were collected and identified according to the phenotypic methods (11). The antibiotic susceptibility of isolates against 13 common antibiotics was investigated using Kirby-Bauer standard disc diffusion method according to CLSI 2011 and 2013 Guidelines. Resistance to methicillin was assessed using cefoxitin disk $(30 \mu \mathrm{g})$ based on CLSI criteria (2013). Furthermore, the presence of $m e c A$ gene was also investigated. DNA of all isolates were extracted using boiling method.

A $S$. aureus strain containing $m e c A$ gene was also extracted and used as positive control. In a multiplex PCR reaction mecA and $p v l$ gene screening was performed; mecAl-F (GTAGAAATGACTGAACGTCCGATAA) and mecA2-R (CCAATTCCACATTGTTTCGGTCTAA) primer pairs and Luk-PV-1F (ATCATTAGGTAAAATGTCTGGACATGATCCA) and Luk-PV2R (GCATCAAGTGTATTGGATAGCAAAAGC) primer pairs were used for amplification of mecA and $p v l$ gene, respectively (12).

The PCR reaction was performed at $25 \mu \mathrm{L}$ final volume containing $12.5 \mu \mathrm{L}$ of $2 \times$ master mix (Master Mix Ampliqon, Denmark), $0.24 \rho \mathrm{M}$ of mecA primers, $0.2 \rho \mathrm{M}$ of Luk-PV primers and $2 \mu \mathrm{L}$ of extracted DNA. The amplification program was: initial denaturation $\left(94^{\circ} \mathrm{C}, 10 \mathrm{~min}\right), 10$ cycles each containing denaturation $\left(94^{\circ} \mathrm{C}, 45 \mathrm{~s}\right)$, annealing $\left(55^{\circ} \mathrm{C}, 45 \mathrm{~s}\right)$ and extension $\left(72^{\circ} \mathrm{C}, 75 \mathrm{~s}\right)$, then 25 cycles were performed as previously mentioned except $50^{\circ} \mathrm{C}$ was used as annealing temperature and a final extension $\left(72^{\circ} \mathrm{C}, 10 \mathrm{~min}\right)$. A negative control (distilled water) and a positive control (mecA positive strain) were also used in parallel. Finally, amplification of $m e c A$ gene (310bp) and $p v l$ gene (433bp) was confirmed through electrophoresis in $1 \%$ agarose containing DNA safe stain. MRSA strains were subjected to SCCmec typing according to the multiplex PCR method described by Zhang et al. (2005) with minor modifications (13).

In order to prevent non-specific reactions, 3 different sets of multiplex PCR reactions were adjusted, each containing 3 primer pairs including set 1: SCCmec type I, IVa and IVb primer pairs; set 2: SCCmec type II, III and V primer pairs, and set 3: SCCmec type I, IVc and IVd. Each $25 \mu \mathrm{L}$ reaction consists in $12.5 \mu \mathrm{L}$ of $2 \times$ Master Mix, 1.5 U Taq DNA polymerase enzyme, $1.5 \mathrm{mM} \mathrm{MgCl}$, $0.4 \rho \mathrm{M}$ of each primer and $5 \mu \mathrm{L}$ of extracted DNA. Amplification program was as follows: initial denaturation $\left(94^{\circ} \mathrm{C}, 15 \mathrm{~min}\right), 10$ cycles consisting in denaturation $\left(94^{\circ} \mathrm{C}, 30 \mathrm{~s}\right)$, annealing $\left(55^{\circ} \mathrm{C}\right.$, $90 \mathrm{~s})$ and extension $\left(72^{\circ} \mathrm{C}, 90 \mathrm{~s}\right)$, then 25 cycles including denaturation $\left(94^{\circ} \mathrm{C}, 45 \mathrm{~s}\right)$, annealing $\left(50^{\circ} \mathrm{C}, 90 \mathrm{~s}\right)$ and extension $\left(72^{\circ} \mathrm{C}, 90 \mathrm{~s}\right)$ and a final extension $\left(72^{\circ} \mathrm{C}, 10 \mathrm{~min}\right)$.

The successful amplification was confirmed through electrophoresis on $1.3 \%$ agarose gel. The data were analysed using SPSS V.17 and Fisher and Chi- square at 0.05 confidence interval.

\section{Results}

Out of the $100 \mathrm{~S}$. aureus strains, 56 were from male patients and 44 from female. The distribution of isolates among clinical specimens was as follows: wound (78), blood (6), tracheal secretions (8), urine (7) and knee abscess (1). In antibiotic susceptibility analysis of MRSA, $58 \%$ of isolates were resistant to cefoxitin, while $97 \%$ and $82 \%$ of strains were resistant to methicillin and oxacillin, respectively. According to the CLSI 2013 Guidelines, the resistance to cefoxitin was regarded as MRSA. In MRSA screening based on mecA amplification, 58 samples were also positive. Therefore, based on the phenotypic and genotypic analysis, the frequency of MRSA was $58 \%$; 60 and $40 \%$ of MRSA were isolated from male and female patients, respectively. The frequency of MRSA based on clinical samples was as follows: wound: $77.5 \%$; tracheal secretions: $5.1 \%$; urine: $6.7 \%$; blood: $8.6 \%$ and knee abscess: $1.7 \%$.

The antibiotic susceptibility profile of all $S$. aureus isolates has been described in Table 1. As it reported, the highest sensitivity

Table 1. Antibiotic susceptibility testing of $S$. aureus isolates.

\begin{tabular}{lccc}
\hline Antimicrobial Agent & Sensitive, $\mathbf{n}(\%)$ & Intermediate, $\mathbf{n}(\%)$ & Resistance, $\mathbf{n}(\%)$ \\
Penicillin (P) & $5(5)$ & - & $95(95)$ \\
Oxacillin (OX) & $17(17)$ & $1(1)$ & $82(82)$ \\
\hline Methicillin (Me) & $2(2)$ & $1(1)$ & $97(97)$ \\
Co-Trimoxazol (SXT) & $31(31)$ & $2(2)$ & $67(67)$ \\
\hline Vancomycin (V) & $48(48)$ & - & $52(52)$ \\
Ciprofloxacin (CP) & $22(22)$ & $9(9)$ & $69(69)$ \\
\hline Erythromycin (E) & $9(9)$ & $7(7)$ & $84(84)$ \\
Chloramphenicol (C) & $59(59)$ & $7(7)$ & $34(34)$ \\
\hline Clindamycin (CC) & $20(20)$ & - & $80(80)$ \\
Gentamycin (GM) & $28(28)$ & $4(4)$ & $68(68)$ \\
\hline Rifampicin (RA) & $34(34)$ & $1(1)$ & $65(65)$ \\
Ceftazidime (CAZ) & $7(7)$ & - & $93(93)$ \\
\hline Imipenem (IPM) & $31(31)$ & $9(9)$ & $60(60)$
\end{tabular}


was to chloramphenicol (59\%), and rifampicin (34\%) and the highest resistance was to methicillin (97\% and penicillin (95\%). Table 2 reports the antibiotic susceptibility of MRSA strains. The highest resistance was to methicillin $(98.27 \%)$, penicillin $(95 \%)$ and the highest sensitivity was to chloramphenicol (36.2\%).

In this study, $82 \%$ of $S$. aureus isolates showed multiple drug resistance (MDR) i.e non-susceptible to oxacillin and at least other 2 antibiotics. Precisely, eight isolates from 10 CA-MRSA and 25 isolates from 27 HA-MRSA had multiple drug resistance. Furthermore, 18 isolates of 21 multiband and non-typeable MRSA showed MDR pattern. Therefore, in total $87.9 \%$ of MRSA had multiple drug resistance. The frequency of MDR in clinical sam- ples was as follows: wound $(76.9 \%)$, tracheal secretions $(62.5 \%)$ and blood, urine and pus (100\%).

The result of multiplex PCR for mecA and $p v l$ revealed that their frequency was $58 \%$ and $4 \%$, respectively. The SCCmec typing of 58 MRSA showed that 5 isolates, including 5,9,13,4 and 6, belonged to SCCmec type I, II, III, IVb and V, respectively (Figure 1). The SCCmec type III was the most frequent type, followed by type II, V, I and IVb.

Three isolates had produced several bands, while SCCmec typing of 18 isolates was impossible, thus indicating that typing was inconclusive and that likely they belong to types VI -XI (14). According to the typing results, we have identified 27 HA-MRSA

Table 2. Antibiotic susceptibility pattern of MRSA.

\begin{tabular}{lccc}
\hline Antimicrobial Agent & Sensitive, $\mathbf{n}(\%)$ & Intermediate, n (\%) & Resistance, n (\%) \\
Penicillin (P) & $3(5)$ & - & $55(95)$ \\
Oxacillin (OX) & $6(10.34)$ & $1(1.72)$ & $51(87.93)$ \\
\hline Methicillin (Me) & $1(1.7)$ & - & $57(98.27)$ \\
Co-Trimoxazol (SXT) & $15(25.86)$ & - & $43(74.13)$ \\
\hline Vancomycin (V) & $28(48.27)$ & $1(1.72)$ & $30(51.72)$ \\
Ciprofloxacin (CP) & $15(25.86)$ & $3(5.17)$ & $42(72.41)$ \\
\hline Erythromycin (E) & $5(8.62)$ & $3(5.17)$ & $50(86.2)$ \\
Chloramphenicol (C) & $21(36.2)$ & - & $34(58.62)$ \\
\hline Clindamycin (CC) & $10(17.24)$ & $1(1.72)$ & $48(82.75)$ \\
Gentamycin (GM) & $13(22.41)$ & $1(1.72)$ & $44(75.86)$ \\
\hline Rifampicin (RA) & $15(25.86)$ & - & $42(72.41)$ \\
Ceftazidime (CAZ) & $5(8.62)$ & $3(5.17)$ & $53(91.37)$ \\
\hline Imipenem (IPM) & $17(29.31)$ & $38(65.51)$ \\
\hline
\end{tabular}

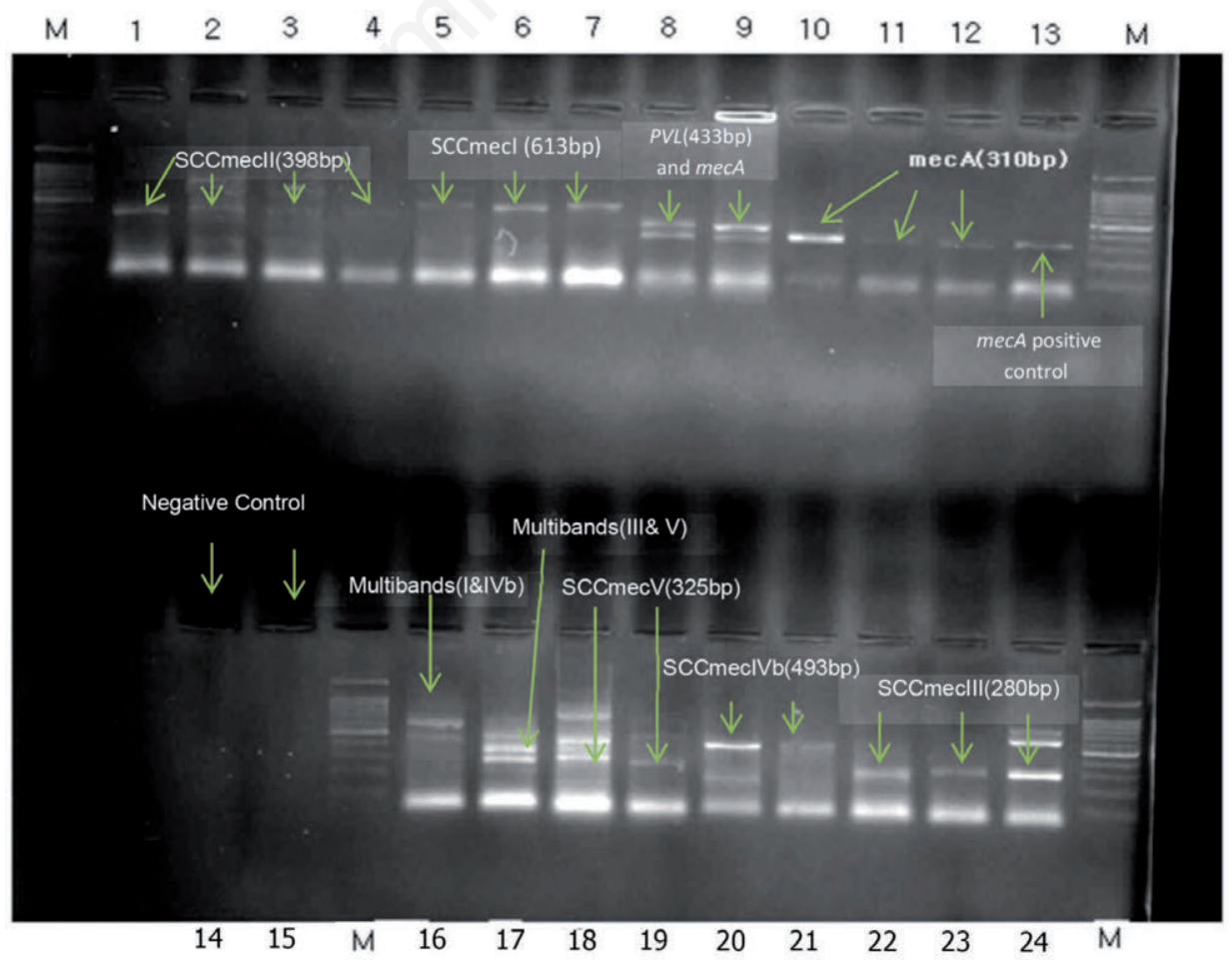

Figure 1. The electrophoresis of PCR products from SCCmec typing and mecA and pvl screening. 
and $10 \mathrm{CA}-\mathrm{MRSA}$ isolates. All $4 \mathrm{pvl}$ positive isolates were categorized as CA-MRSA; more precisely, 3 isolates of type $\mathrm{V}$ and 1 isolate of type $\mathrm{IVb}$. These results are in agreement with previous findings indicating that $p v l$ positive MRSA belong to type IV and V and are related to CA-MRSA. Therefore, the presence of $p v l$ can be regarded as a genetic marker for CA-MRSA (4). Table 3 presents the frequency of SCCmec types among MRSA.

The frequency of SCCmec types based on the clinical samples is reported in Table 4. The most common type, i.e. type III, was isolated from wound specimens.

Statistical analysis shows that there is a significant $\left(\chi^{2}<0.001\right)$ correlation between phenotypic methicillin resistance and mecA in these isolates. Furthermore, the multiple drug resistance is in correlation $\left(\chi^{2}=0.020\right)$ with the presence of $m e c A$. A significant relation was found between the CA-MRSA and $p v l$ gene $(\mathrm{P}<0.001)$, and also between the urine samples with the presence of HA$\operatorname{MRSA}(\mathrm{P}=0.041)$.

\section{Discussion}

With regard to the constant increase of antibiotic resistance in pathogenic bacteria and the consequent problems in health care systems, monitoring of antibiotic resistance pattern of local pathogens is of great importance in order to prevent antibiotic treatment failure of infectious diseases. Furthermore, using suitable genetic markers for finding the source of infections can help clinicians to diminish or even prevent infections. Therefore, the present study has evaluated antibiotic resistance pattern of $S$. aureus isolates from a Hospital in Ahvaz. The results showed that resistance to methicillin is the high- est resistance pattern while the least resistance was to chloramphenicol, as reported by Rezazadeh et al. (15). In the study of Moradi et al., the majority of isolates was sensitive to vancomycin, chloramphenicol and rifampicin, and the highest resistance was found against cefoxitin (16).

In this study, the frequency of MRSA was $58 \%$, of which $46.5 \%$ categorized as HA-MRSA and $17.2 \%$ as CA-MRSA, highlighting the prevalence of HA-MRSA. This finding is in agreement with similar studies carried out in China, Australia and Africa (5$25 \%$ range), Portugal (49\%), Greece (40\%), Italy (37\%) and Romania (34\%) (17). Fathollah Zadeh et al., in their study carried out in Tehran have reported a MRSA frequency of $36 \%$ (18), while Khosravi et al. showed a prevalence of $87.3 \%$ (19), Zeinali et al. 58\% (6), and Abdollahi et al. 47.5\% (20), respectively. The differences between the reported data from different regions can be a result of the different infection control programs, antibiotic therapy regimens and other factors that can affect resistance spread among bacterial species.

The most frequent SCCmec type in the present study was SCCmec type III. Similarly, among the HA-MRSA, SCCmec type III has been the dominant type in Taiwan, Hong Kong, Thailand, India and Srilanka (21).Conversely, Zeinali et al. (2011) reported that SCCmec type II was the most frequent type and none of the MRSA strains had SCCmec type III. Furthermore, 58.6\% of their samples were non-typeable (6). Similarly, in the study of Abdollahi et al. (2012), SCCmec type II was the most frequent (20). Prevalence of five SCCmec types in the present study proves the high variety of types in the area under study.

The prevalence of $p v l$ gene in MRSA isolates was $4 \%$, all of them belong to CA-MRSA. Different studies have reported frequency values from 2 to $35 \%(22,23)$, while in Argentina and Iran

Table 3. SCCmec type frequency among MRSA.

\begin{tabular}{lccc} 
SCCmec type & CA-MRSA/HA-MRS & Amplicon sive (bp) & N. of isolates (\%) \\
SCCmecI & HA-MRSA & 613 & $5(8.62)$ \\
SCCmecII & HA-MRSA & 398 & $9(15.51)$ \\
\hline SCCmecIII & HA-MRSA & 280 & $13(22.41)$ \\
SCCmecIVa & CA-MRSA & 776 & - \\
\hline SCCmecIVb & CA-MRSA & 493 & $4(6.89)$ \\
SCCmecIVc & CA-MRSA & 200 & - \\
\hline SCCmecIVd & CA-MRSA & 881 & - \\
SCCmecV & CA-MRSA & 325 & $6(10.34)$ \\
\hline Multiple bands SCCmec III and SCCmec IVb & Unidentified & $(280+493)$ & $1(1.72)$ \\
Multiple bands SCCmec I and SCCmec IVb & Unidentified & $(613+493)$ & $1(1.72)$ \\
\hline Multiple bands SCCmec III and SCCmec V & Unidentified & $(280+325)$ & $1(1.72)$ \\
Non typable & & & $18(31.03)$ \\
\hline
\end{tabular}

Table 4. Frequency of SCCmec types in different clinical specimens.

\begin{tabular}{|c|c|c|c|c|c|c|c|c|c|c|}
\hline & SCCmecI & scCmecII & SCCmeclII & scCmecIVa & SCCmecIVb & SCCmecIVc & scCmecIVd & scCmecV & Multibands & Nontypable \\
\hline Wound (45) & 4 & 6 & 11 & - & 3 & - & - & 5 & 1 & 15 \\
\hline Blood (5) & - & 1 & - & - & - & - & - & 1 & 2 & 1 \\
\hline Urine (4) & 1 & 2 & 1 & - & - & - & - & - & - & - \\
\hline Tracheal secretions (3) & ） - & - & - & - & 1 & - & - & - & - & 2 \\
\hline Knee Abscess (1) & - & - & 1 & - & - & - & - & - & - & - \\
\hline Total 58 & 5 & 9 & 13 & - & 4 & - & - & 6 & 3 & 18 \\
\hline
\end{tabular}


have been observed values of $56 \%$ and $61.8 \%$, respectively. These differences can be related to the frequency of this gene, as well as to the different methodologies that have been used.

The isolates from skin, wound and pulmonary infections showed a higher prevalence than other clinical samples $(24,25)$. Interestingly, all four $p v l$ positive isolates in this study originated from wound samples. In the report of Khosravi et al. in burn patients of Ahvaz, $87 \%$ of isolates had mecA gene and $7 \%$ were positive for $p v l$ (19). Abdorrazagh et al. (2014), have reported a $27 \%$ prevalence of $p v l$ in Iraq (26). Shore et al. found that the frequency of $p v l$ in MRSA isolates during 2002-2011 increased from 2 to $8.8 \%$, while its frequency in MSSA decreased from 20 to $2.5 \%$ (27). The cross-sectional study results of Qiwen et al. on $259 \mathrm{HA}-$ MRSA showed a $p v l$ prevalence of $28.6 \%$ (28).

\section{Conclusions}

High genotypic diversity of MRSA in the present study in Ahvaz may be a result of population diversity, as well as its borderline geographical location. All SCCmec types in this study showed a multiple drug resistance. This fact can lead to both treatment failure in patients and increases in the costs for infection control. This finding can be a serious threat for the health care management system in order to correctly use antibiotics for infection control. With regard to the dangerous nature of $p v l$ positive $S$. aureus, the infections caused by these isolates can be a life-threatening factor. Therefore, early diagnosis and treatment of these infections are recommended. The results of this study suggest that by using PCR technique it is possible to detect multiple resistant strains and their SCCmec types and, thus, control their origin. Finally, it can be hypothesized that, based on the significant correlation between $p v l$, SCCmec and CA-MRSA, it is necessary that screening of $S$. aureus isolates be regarded as a useful approach for the diagnosis of these strains and their control.

\section{References}

1. Amini D, Keshavarzi F, Fatahi Rad A. Comparison phenotypic methods with mecA gene analysis for the detection of methicillin/oxacillin resistance in clinical isolates of Staphylococcus aurous in Sanandaj city. SJIMU 2014;22:109-22.

2. Alfatemi SM, Motamedifar M, Hadi N, et al. Analysis of virulence genes among methicillin resistant Staphylococcus aureus (MRSA) strains. Jundishapur J Microbiol 2014;7:1-10.

3. Conceicao T, Coelho C, Santos-Silva I, et al. Epidemiology of Methicillin-Resistant and-Susceptible Staphylococcus aureus in Luanda, Angola: First Description of the Spread of the MRSA ST5-Iva Clone in the African Continent. Microb Drug Resist 2014;20:441-9.

4. Mack D, Becker P, Chatterjee I, et al. Mechanisms of biofilm formation in Staphylococcus epidermidis and Staphylococcus aureus: functional molecules, regulatory circuits, and adaptive responses. Int J Med Microbiol 2004;294:203-12.

5. Szabo J. Molecular Methods in Epidemiology of Methicillin Resistant Staphylococcus aureus (MRSA): Advantages, Disadvantages of Different Techniques. J Med Microb Diagn 2014;3:147.

6. Zeinali E, Moniri R, Safari M, et al. Molecular characterization and SCCmec typing in meticillin-resistant Staphylococcus aureus isolated from clinical samples. Feyz 2011;14:439-46.
7. Smith CS, Parnell P, Hodgson G, et al. Are methicillin-resistant Staphylococcus aureus that produce Panton-Valentine leucocidin (PVL) found among residents of care homes? J Antimicrob Chemother 2008;62:968-72.

8. Vinodhkumaradithyaa A, Uma A, Shirivasan M, et al. Nasal Carriage of methicillin-resistant Staphylococcus aureus among surgical unit staff. Jpn J Infect Dis 2009;62:228-9.

9. Yanagihara K, Kihara R, Araki N, et al. Efficacy of linezolid against Panton Valentine leukocidin-positive methicillin-resistant Staphylococcus aureus (MRSA) in a mouse model of haematogenous pulmonary infection. Int J Antimicrob Agents 2009;34:477-81.

10. Kim SJ, Park C. Panton-Valentine Leukocidin and Staphylococcal Cassette Chromosome (SCCmec) from CAMRSA (Community-Acquired Methicillin Resistanct Staphylococcus aureus). Biomed Res 2014;25:441-4.

11. Murray PR, Boriello P, eds. Topley and Wilson's Microbiology and Microbial infection. 10th ed. London: Hodder Arnold; 2005.

12. McClure J, Conly JM, Lau G. Novel multiplex PCR assay for detection of the staphylococcal virulence marker PantonValentine leukocidin genes and simultaneous discrimination of methicillin-susceptible from resistant staphylococci. J Clin Microbiol 2006;44:1141-4.

13. Zhang K, McClure JA, Elsayed S, et al. Novel multiplex PCR assay for characterization and concomitant subtyping of staphylococcal cassette chromosome mec types I to V in methicillin-resistant Staphylococcus aureus. J Clin Microbiol 2005;43:5026-33.

14. Makgotlho PE, Kock MM, Hoosen A, et al. Molecular identification and genotyping of MRSA isolates.Department of Medical Microbiology, University of Pretoria/NHLS, Pretoria, South Africa. FEMS Immunol Med Microbiol 2009;57:104-15

15. Rezazadeh M, Yousefi Mashouf R, et al. Antibiotic Profile of Methicillin-Resistant Staphylococcus Aureus With MultipleDrug Resistances Isolated from Nosocomial Infections in ValiAsr Hospital of Arak. AMUJ 2013;16:29-37

16. Moradi N, Javadpour S, Karmostaji A. Reduced sensitivity of staphylococcus aureus to vancomycin. HMJ 2011;15:169-77.

17. Stefani S, Chung DR, Lindsay JA, et al. Meticillin-resistant Staphylococcus aureus (MRSA): global epidemiology and harmonisation of typing methods. Int $\mathrm{J}$ Antimicrob Agents 2012;39:273-82.

18. Fatholahzadeh B, Emaneini M, Gilbert G, et al. Staphylococcal Cassette Chromosome mec (SCCmec) Analysis and Antimicrobial Susceptibility Patterns of Methicillin-Resistant Staphylococcus aureus (MRSA) Isolates in Tehran, Iran. Microbial Drug Resistance 2008;14:217-20.

19. Khosravi AD, Hoveizavi H, Farshadzadeh Z. The prevalence of genes encoding leukocidins in Staphylococcus aureus strains resistant and sensitive to methicillin isolated from burn patients in Taleghani hospital, Ahvaz, Iran. Burns 2012;38:247-51.

20. Abdollahi A, Kouhpaye S, Najafipour S, et al. Evaluation of drug resistance and staphylococcal chromosomal cassette mec (SCCmec) genotype in Methicillin-Resistant Staphylococcus aureus strains. AUMJ 2012;1:47-52.

21. Song JH, Hsueh PR, Chung DR, et al. Spread of methicillinresistant Staphylococcus aureus between the community and the hospitals in Asian countries: an ANSORP study. J Antimicrob Chemother 2011;66:1061-9.

22 Melles DC, Gorkink RF, Boelens HA, et al. Natural population dynamic and expansion of pathogenic clones of Staphylococcus aureus. J Clin Invest 2004;114:1732-40. 
23. Santucci Z, Gobara S, Santos CR, et al. Infections in a burn intensive care unit: experience of seven years. J Hosp Infect 2003;53:6-13.

24. Havaei SA, Ohadian Moghadam S, Pourmand MR, et al. Prevalence of genes encoding bi-component leukocidin among clinical isolates of methicillin-resistant Staphylococcus aureus. Iran J Publ Health 2010;39:8-14.

25. Holmes A, Ganner M, McGuane S, et al. Staphylococcus aureus isolates carrying Panton-Valentine Leucocidin genes in England and Wales: frequency, characterization, and association with clinical disease. J Clin Microbiol 2005;43:2384-90.

26. Abdulrazaq RA, AL-Marjaniand MF, Othman SH. Prevalence of genes encoding Exfoliatin toxin A and Panton-Valentine
Leukocidin among Methicillin resistant Staphylococcus aureus in Baghdad. Int J Curr Microbiol Appl Sci 2014;3:595-600.

27. Shore AC, SC, Brennan GI, et al. Panton-Valentine Leukocidin-Positive Staphylococcus aureus in Ireland from 2002 to 2011: 21 Clones, Frequent Importation of Clones, Temporal Shifts of Predominant Methicillin-Resistant S. aureus Clones, and Increasing Multiresistance. J Clin Microbiol 2014;52:859-70.

28. Hu Q, Cheng H, Yuan W, et al. Panton-Valentine Leukocidin (PVL)-positive Healthcare-associated Methicillin-resistant Staphylococcus aureus Isolates Are Associated with Skin and Soft Tissue Infections and Colonized Mainly by Infective PVLencoding Bacteriophages. J Clin Microbiol 2015;53:67-72. 